\title{
DRIVER ALERTNESS DETECTION RESEARCH USING CAPACITIVE SENSOR ARRAY
}

\author{
Philip W. Kithil \\ Advanced Safety Concepts, Inc. \\ 1570 Pacheco Street, Suite E-12 \\ Santa Fe, NM 87505 \\ Email: pkithil@headtrak.com
}

\section{Summary}

The research project compared and analyzed physiological and performance data for 13 subjects driving a vehicle simulator. Each subject drove the simulator for morning, afternoon, and late night sessions. These sessions were intended to represent alertness conditions during an "awake" baseline period and the secondary and primary circadian sleep cycle periods. The sessions were approximately one hour, two hours, and two or three hours in length, respectively. With one exception, the subjects had experienced normal sleep the night before the test. Five men and eight women participated, ranging in age from 25 to 59.

Physiological data included: real-time PERCLOS (percentage of slow-eye closure over one minute) using an infrared-reflective camera; head position coordinates using an overhead capacitive sensor array; and video of the right front of the subject's face. Performance data included: vehicle speed, lane departures, lane deviation, and steering/turn signal data. The research manager maintained logs of unusual circumstances such as departing the roadway, falling asleep at the wheel, excessive speeding, etc.

Head position data was analyzed and compared to the videos. A multi-element algorithm was developed which captured patterns of head motion found to be characteristic of drowsiness. The algorithm output was compared to roadway departures noted in the research manager's logs of unusual events. The comparison showed a capability of advance detection of about $87 \%$ of driver roadway departures with a false positive rate of about $15 \%$.

\section{INTRODUCTION}

It is estimated that nearly 8,000 highway fatalities each year are directly or indirectly caused by driver inattention, fatigue, or sleepiness, based on the most recent Fatal Accident Reporting System (FARS) data compiled by National Highway Traffic Safety Administration. A low-cost system that could detect driver alertness/sleepiness offers significant potential to save thousands of lives each year. 


\section{BACKGROUND}

The author's SAE Paper 982292 “Capacitive Occupant Sensing” (Kithil, 1998) presented at International Body Engineering Conference, September 29-October 1, 1998, describes various applications of an adjacent-electrode capacitive sensor under development by Advanced Safety Concepts, Inc. (ASCI). The paper covers headliner, airbag door, and windshield-embedded sensors. The sensor physics relies on the shielding of sensor electrode mutual capacitance by the highly conductive human body. When a person enters the capacitive field, this shielding effect results in a decrease in the mutual capacitance. The change in capacitance correlates to proximity and is described by a $1 / \mathrm{r} 2$ relationship. With three sensors providing overlapping fields beneath the headliner, it is possible to triangulate head position. Head position "XYZ" coordinates may be used for various functions including airbag control, detecting driver "nodding off", to adjust headrest position, mirror viewing angle, etc.

Unpublished 1995 research by University of New Mexico Hospital Sleep Disorders Center used the ASCI capacitive sensor array to quantify changes in head position while sleep-deprived State Highway Department snow plow drivers attempted to perform a computer driving task. Some correlation was apparent between very small changes in head position ("micro-nods") and "micro-sleep" episodes. The micro-sleep episodes were determined by EEG/EOG strip charts, which were analyzed by a sleep technician. A micro-sleep episode is a momentary period of sleep, which often precedes a deeper sleep condition. Conclusive analysis of the correlation was not performed due to funding limitations.

In 1996 and 1997, the Capacitive sensor array was supplied to Carnegie Mellon Research Institute and to University of Pennsylvania Department of Psychiatry for several sleep research experiments funded by U.S. Department of Transportation - Federal Highway Administration Office of Motor Carrier Safety. The basis for including the Capacitive sensors in these experiments was to allow the researchers to develop a better understanding of the capabilities and to initiate some comparison between the sensing system and other drowsiness-detection technologies.

These other technologies included an eye-closure detection system, which had demonstrated drowsiness-detection capabilities. Termed "PERCLOS", the device and detection techniques relied on the percentage of slow-eyelid closures during a one to three minute period.

Subsequently, PERCLOS has been "validated" as a measure of drowsiness based on favorable correlation of PERCLOS scores with psychomotor vigilance test (PVT) scores, both withinsubjects and between-subjects. PVT has been shown to be a reliable indicator of decreased alertness (Dinges, 1999). The validation tests were conducted in University of Pennsylvania's sleep-research laboratory using computer-generated simulations of a vehicle operating on an interstate highway. In these tests, the ASCI system demonstrated very high correlation for some test subjects and very low correlation for others. ASCI attributes these uneven results to equipment problems, the laboratory setup, and the unrealistic test conditions.

In 1999, ASCI was able to obtain private funding for a pilot drowsy driver study using its sensors compared to a sophisticated EEG/EOG-based detection technique. Four subjects were kept awake all night then drove a computer simulator in a research laboratory for several hours. The 
head motion data showed significant correlation to the EEG/EOG-scored indications of drowsiness.

\section{PROJECT DESCRIPTION}

Based on these results, the funding source agreed to sponsor more comprehensive research using a larger sample group in a vehicle simulator. The TruckSim ${ }^{\circledR}$ facility at Carnegie Mellon’s National Robotics Engineering Consortium (NREC) facility was used. Under the direction of NREC’s Richard Grace, Ph.D., over 60 subjects were interviewed and about 20 selected and screened for participation in the study.

The study specified three driving bouts in the simulator: $11 \mathrm{am}$ to noon; 3pm to 5pm; and 3am to 5am. This design was intended to capture data for a normal "awake” period, a period encompassing the semi-circadian sleep cycle, and a period encompassing the late night circadian sleep cycle. Circadian cycle refers to our inherent biological urge to sleep during specified time periods - particularly mid-afternoon and at night. Subjects were instructed to obtain normal sleep preceding the day of the test. Actigraphs, a wrist-based sleep monitoring device, were provided to allow verification of the previous 24 hours sleep. PVT tests were administered before and after each bout, and at 2 hour intervals between bouts.

The TruckSim offers a 150 degree field of view of a simulated divided highway environment. The highway repeats about every 20 miles but road signs are changed to give it the appearance of "new" roadway. The simulator was configured to drive like a mid-size US passenger car, except for the seat, steering wheel, and side mirrors, which retained truck-like characteristics. The simulator motion base was not used, to give the "feel" of a smooth car ride.

Real-time data obtained included:

1. Eye coordinates and eye closure obtained from an infrared camera using retinal reflectance.

2. PERCLOS, a one-minute calculation of slow eye closures, using $>80 \%$ eye closure (pupil obscured).

3. Sensor voltages from the ASCI overhead capacitive sensor array.

4. Video showing the right front of the driver's face.

5. Simulator data (speed, lane tracking, wheel angle, turn signal use, etc.).

The sensor voltages were post-processed by ASCI to derive head position XYZ coordinates. Simulator data was post-processed by the simulator facility to derive relevant vehicle data, particularly one-minute averages of speed, lane departures, and lane deviations. The researcher maintained logs of significant driver or simulator events such as the subject's PERCLOS level, signs of wakefulness or drowsiness (yawns, etc.), and noteworthy vehicle simulator events (swerving, speeding, and the like). These logs were not part of the original research protocol but became an important supplement to the primary data stream.

After the ASCI drowsiness detector was developed, its output was compared to PERCLOS. Thresholds were selected to allow a determination if either detector provided advance detection of driving "errors" noted in the simulator logs kept by the research manager. 
Ultimately, useable data was captured for 13 subjects - 5 men and 8 women. Age ranged from 25 to 59. A total of 74 hours of data was acquired.

Staff from ASCI, Complexica, Inc., and Mesa Analytics \& Computing LLC, analyzed the driver data, logs kept by the simulator manager of each driver's critical driving errors (running off the road events, etc.) as well as the videos and simulator data itself. From this an early warning detector of dangerous drowsy driving behavior was developed. This detector was designed to detect dangerous drowsy driver behavior in signals from the ASCI capacitive sensor array. The signals are strongly correlated with the head position of the driver. The software detector extracts head motion behavior associated with driving while drowsy. The detector is adaptive in that it learns the normal characteristics of individual driver and is able to identify the drowsy behavior patterns of each individual.

The performance of the detector was compared to PERCLOS. Ten of the 13 drivers experienced a total of 38 major driving errors (e.g. departing the roadway), according to logs maintained by the project manager. The head motion system provided advance detection of $100 \%$ of these "errors" for 7 of the 10 drivers, versus PERCLOS which provided 100\% advance detection of these "errors" for 3 of the 10 drivers. Overall, the head motion system predicted $87 \%$ of all driving “errors", vs. 34\% for PERCLOS. The average false positive rate for the head motion system was $14.9 \%$ versus $9.2 \%$ for PERCLOS. It should be noted the ASCI Detection method used a personalized threshold to indicate a subject's impairment due to drowsiness, derived from the morning "awake” driving session, whereas the PERCLOS detection method used a generalized threshold to indicate a subject's impairment due to drowsiness. Two threshold values were tried for the PERCLOS method - 8\% and 12\%, with the lower value (as expected) generating a higher detection rate and higher false positives.

The standard used in comparing the ASCI detector and PERCLOS was to determine predictability of the logged driving "errors" during a four-minute period preceding the minute of the driving "error". This was the best available method because the logs did not specify the exact time of the "error". If either detector was above its threshold at any time during the four minute period this was counted as a "detection" which could have activated some form of warning to the driver, and possibly have prevented the driving "error".

Researchers used a training sample of 10 drivers to tune the ASCI detector and a testing sample of 3 drivers in order to confirm that they were not over fitting the detector. Since the entire data set consisted of only 13 drivers, no further cross-validation of the detector was attempted (e.g., various forms of $k$-fold cross-validation methods).

\section{CONCLUSION}

The data show that the capacitive sensor head motion detector may be a more robust method of detecting drowsiness, compared to currently-available technologies such as PERCLOS. Results are based on driving simulation data with thirteen drivers, where all drivers were tested both during awake and sleep deprived conditions. From observing videotapes of the drivers in the driving simulator, the researchers felt that PERCLOS suffers from the variability of individual driving and sleep deprivation behavior. Some drivers clearly fall asleep or have drowsy episodes 
with their eyes open or more widely open than others. PERCLOS as a measure is also confounded by a high false positive rate due to the inability of the camera to track the eyes when the driver's head moves out of range for some reason (glances, readjusting seat position, leaning forward, etc.). The capacitive sensor head motion detector, which uses a collection of head position features over different time intervals, seems to be a more sensitive measure of driver drowsiness than PERCLOS.

\section{ACKNOWLEDGEMENTS}

The author gratefully acknowledges the financial support of Johnson Controls, Inc. In addition, the involvement of Roger Jones, Ph.D. of Complexica, Inc., and John MacCuish, Ph.D. of Mesa Analytics \& Computing LLC in data analysis and algorithm development has been instrumental in attaining the results shown.

\section{REFERENCES}

1. Dinges, David F., Ph.D., Grace, Richard, Ph.D. PERCLOS: A Valid Psychophysiological Measure of Alertness As Assessed by Psychomotor Vigilance. Federal Highway Administration, Office of Motor Carriers, 1998.

2. Kithil, Philip W., SAE Technical Paper Series 982292, Capacitive Occupant Sensing, Proceedings from the IBEC '98, Volume 6 Safety, Environmental, and Automotive Interior Systems (P-335), SAE International, 1998. 